\title{
Enhanced aquaporin 8 expression after subtotal colectomy in rat
}

\author{
Masato Nakano ${ }^{1}$, Yu Koyama ${ }^{1 *}$, Hitoshi Nogami ${ }^{1}$, Tadashi Yamamoto ${ }^{2}$, Toshifumi Wakai ${ }^{1}$ \\ ${ }^{1}$ Division of Digestive \& General Surgery, Niigata University Graduate School of Medical \& Dental Sciences, Niigata, Japan \\ ${ }^{2}$ Division of Renal Pathology, Niigata University Graduate School of Medical \& Dental Sciences, Niigata, Japan \\ Email: *yukmy@med.niigata-u.ac.jp
}

Received 11 July 2013; revised 12 August 2013; accepted 26 August 2013

Copyright (c) 2013 Masato Nakano et al. This is an open access article distributed under the Creative Commons Attribution License, which permits unrestricted use, distribution, and reproduction in any medium, provided the original work is properly cited.

\begin{abstract}
Background: Aquaporins (AQPs), the family of water-selective channels, are localized in various organs and tissues, including the gastrointestinal (GI) tract. However, the roles of AQPs in the GI tract remain unclear. Materials and Methods: Male SD rats were subjected to subtotal colectomy (Group $C, \mathbf{n}=22$ ) or a sham operation (Group $S, n=16$ ) and were sacrificed on postoperative days 7,14 , and 28 . Total RNAs from the distal ileum and rectum were extracted. Quantitative RT-PCR was performed to measure AQP8 mRNA expression. For light-microscopy or immunohistochemistry, paraffin-embedded sections of 4 $\mu \mathrm{m}$ were prepared with H-E staining or anti-AQP8 antibody reaction. Mann-Whitney U-test was performed to compare the AQP8 distributions between the two groups, and the statistical significance was defined as $p<0.05$. Results: AQP8 mRNA expression was enhanced in both the ileum and rectum in Group C at day 7. AQP8 protein expression was consistently observed in the ileum and rectum. The villus length in the ileum of Group $C$ was significantly greater than that of Group $S$ at days 7 and 14. Conclusion: Enhanced AQP8 mRNA expression in the subtotal colectomy model suggests that AQP8 plays an important role in maintaining the intestinal fluid balance.
\end{abstract}

Keywords: Aquaporin 8; Subtotal Colectomy; mRNA; Immunohistochemistry

\section{INTRODUCTION}

Water-selective channels (aquaporins; AQPs) have been identified as molecules located mainly on the plasma membrane of various cell types and increasing water permeability. The mammalian water channel, AQP1, was first identified as a protein homologous to the major intrinsic protein of the bovine lens in erythrocytes [1,2], and was also shown to be present in red blood cells, renal proximal convoluted tubules and the thin descending limb of Henle [1,2]. Homology cloning techniques have been used to clone AQPs, which have been cloned from various organs: AQP2 [3] and AQP3 [4] from the kidney, AQP4 from the brain [5], AQP5 from the salivary gland [6], AQP6 from the kidney [7], AQP7 from the testis [8], AQP8 from the pancreas and liver [9], AQP9 from the liver [10], and AQP10 from the duodenum and jejunum [11].

Previous studies have demonstrated the expression of several AQP types in the gastrointestinal (GI) tract, suggesting their participation in water absorption or secretion $[1,4,5,9]$. We have demonstrated both mRNA and protein expression of AQP1, AQP3, AQP4, and AQP8 in the rat GI tract $[12,13]$. However, the functions of the AQPs in the GI tract have not been well elucidated. In the present study, we examined the mRNA expression and protein expression of AQP8 in rat subtotal colectomy models in order to investigate the possible functions of AQP8 in the GI tract during postoperative adaptation to subtotal colectomy.

\section{MATERIALS \& METHODS}

\subsection{Experimental Animals}

All experiments and surgical procedures conformed to the guidelines for the proper care and use of laboratory animals issued by the Public Health Service, National Institutes of Health. Male Sprague-Dawley (SD) rats weighing approximately $200 \mathrm{~g}$ were purchased from Charles River Japan (Yokohama, Japan). Throughout the experimental period, except for $12 \mathrm{~h}$ prior to the surgical procedures, the animals were allowed free access to standard lab rat chow and tap water. Their condition and weight were 
recorded daily.

\subsection{Subtotal Colectomy Model}

The rats were divided into two groups: Group C $(n=22)$ was subjected to subtotal colectomy with primary anastomosis and Group S $(\mathrm{n}=16)$, a sham operation. They were denied food for $12 \mathrm{~h}$ and were anesthetized by intraperitoneal injection of pentobarbital. In Group C, a short segment of the ileum and the entire large intestine was resected from $1 \mathrm{~cm}$ proximal to the ileocecal valve to $2 \mathrm{~cm}$ proximal to the anal verge, and ileoproctal anastomosis was performed. In Group S, the ileum was divided at $1 \mathrm{~cm}$ proximal to the ileocecal valve, and the rectum was divided at $2 \mathrm{~cm}$ proximal to the anal verge. Subsequently, anastomosis was performed without removal of the small or large intestine. All bowel anastomoses were completed with eight interrupted 5-0 silk sutures. The abdominal wound was closed in two running layers, using 2-0 silk. Both groups were allowed free access to standard lab rat chow and tap water after the operation.

The animals in both Group C and Group S were sacrificed at postoperative days 7 ( $\mathrm{n}=8$ and $\mathrm{n}=6$, respectively), $14(n=7$ and $n=5$, respectively), and $28(n=7$ and $n=5$, respectively). Total RNAs were isolated from the ileum and the remnant rectum by a modified acid guanidiniumthiocyanate phenol-chloroform extraction method using TRIzol (GIBCO BRL, Life Technologies, Rockville, MD, USA), as described previously [12].

\subsection{PCR Cloning of Rat Aquaporin 8}

Rat AQP8 (315 bp; +701 +1015) cDNA fragments were obtained from the rat ileum and remnant rectum by the PCR-based cloning method using primers for AQP8 as reported previously $[9,12]$. The PCR products were subcloned into pGEM 11Z (Promega Japan Inc., Tokyo, Japan) and their sequences were verified using an automated DNA sequencer (Perkin Elmer, Foster City, CA, USA). A partial fragment of rat glyceraldehyde-3-phosphate dehydrogenase (GAPDH) cDNA of 123 bp was inserted in pGEM $3 Z$ (Promega).

The plasmid with the rat AQP8 and GAPDH gene inserts was linearized with appropriate restriction enzymes and used as the template for quantitative RT-PCR.

\subsection{Quantitative RT-PCR}

The total RNA ( $1 \mu \mathrm{g})$ of each sample was reverse-transcribed at $42^{\circ} \mathrm{C}$ for $1 \mathrm{~h}$ by using an oligo (dT) primer and Superscript II reverse transcriptase (GIBCO BRL) in a volume of $20 \mu \mathrm{l}$. Each of these transcripts was used as a template for multiplex quantitative PCR to measure AQP8 mRNA and GAPDH expression in a single well by using an ABI PRISM 7700 sequence detection instrument (PE Biosystems Japan, Chiba, Japan).
The AQP8-specific primers were 5'-GGCAGGTGGT GGGATCTCT-3' and 5'-GCCTAATGAGCAGTCCCA CAA-3', and the fluorogenic probe was 5'-TGGATC TACTGGCTGGGCCCACTC-3'. A GAPDH TaqMan Rodent GAPDH Control Reagent VIC TM Probe (PE Biosystems Japan) was used to amplify rat GAPDH for use as an internal control.

The amplification reactions mixture $(50 \mu \mathrm{l})$ contained a reverse-transcript $(1 \mu \mathrm{l}), 1 \times$ TaqMan Universal PCR Master Mix (PE Biosystems), $900 \mathrm{nM}$ of each AQP8 primer, $250 \mathrm{nM}$ of the AQP8 fluorogenic probe, $100 \mathrm{nM}$ of rodent GAPDH primers, and $250 \mathrm{nM}$ of the rodent GAPDH fluorogenic probe. All quantitative 2-step -PCR reactions were performed according to the manufacturer's instructions under the following thermocycler conditions: $50^{\circ} \mathrm{C}$ holding for $2 \mathrm{~min}, 95^{\circ} \mathrm{C}$ holding for $10 \mathrm{~min}$ followed by 40 cycles at $95^{\circ} \mathrm{C}$ for 15 seconds, and at $60^{\circ} \mathrm{C}$ for 1 min. Template-negative controls were run on each PCR plate. A calibrator reverse-transcript sample was amplified in parallel on all plates in order to allow a comparison of the samples run at different times. The data were analyzed using Sequence Detection Software (PE Biosystems Japan), and the values were represented as the mean $(\mathrm{SEM})$ of ratios (AQP/GAPDH mRNA amplicon) $\times$ $100 \%$.

\subsection{Immunohistochemistry}

Since the results of mRNA quantification revealed enhanced AQP8 expression in the ileum and the remnant rectum of the Group $C$ animals, immunohistochemistry was performed as described previously [13]. In brief, the rat ileum and rectum samples were cut and fixed with methyl-Carnoy's fixative (60\% methanol, 30\% chloroform, $10 \%$ acetic acid) overnight, dehydrated with ethanol, embedded in paraffin, and sectioned at $4 \mu \mathrm{m}$. The slide-sectioned tissues were deparaffinated with xylene and ethanol, hydrated in distilled water, and then blocked with normal goat serum (1:20 dilution) for $1 \mathrm{~h}$. After a three-time rinse with PBS, the slides were incubated with an anti-AQP8 antibody $(2.0 \mu \mathrm{g} / \mathrm{ml}$ ) (Alpha Diagnostic Intl. Inc., San Antonio, TX, USA) for $1 \mathrm{~h}$ at $37^{\circ} \mathrm{C}$; this was followed by overnight incubation at $4^{\circ} \mathrm{C}$, rinsing three times with PBS, and incubationed with goat antirabbit immunoglobulins conjugated to a peroxidase-labeled polymer (En Vision, DAKO, Kyoto, Japan), and coloring by diaminobenzidine reaction. After rinsing with distilled water, the slides were counterstained with hematoxylin for observation.

\subsection{Morphologic Measurements}

To measure the villus length of the ileum and the crypt depth of the remnant rectum and the ileum, rat ileum and rectum samples were cut and fixed as described above. 
The slide-sectioned tissues were deparaffinated with xylene and ethanol, hydrated in distilled water, and then immersed in a Hematoxylin-Eosin solution for $15 \mathrm{~min}$. The measurements were performed 5 times in each slide section, and the values were described as the mean \pm SD.

\subsection{Statistical Analysis}

Mann-Whitney U-test was performed to compare the distributions between the two groups. A two-tailed p value of $<0.05$ was considered to indicate statistical significance.

\section{RESULTS}

\subsection{Overall Animal Health}

During the current experimental series, the perioperative lethality was $40 \%$. Twenty-five rats were sacrificed due to bleeding from the mesenterium, ileus or peritonitis. The weight of the Group $\mathrm{C}$ rats decreased and diarrhea was observed until day 7. The weight of the Group S rats decreased until day 3; however, it gradually increased after day 4. Moreover, no diarrhea was observed. The rats in Group C showed a temporary decrease in weight from $210.5 \pm 14.9 \mathrm{~g}$ to $165.8 \pm 20.0 \mathrm{~g}$, 7 days after the operation. The other group showed a temporary decrease from $200.2 \pm 8.2$ g to $192.7 \pm 8.4 \mathrm{~g}$, 3 days after the operation. The weight loss after surgery was more severe in Group C as compared to Group S (Group C: $210.5 \pm 14.9$ to $165.8 \pm 20.0$, Group S: $200.2 \pm 8.2$ to $192.7 \pm 8.4$ ). However, it recovered gradually in both groups, although the postoperative weight on days 7,14 , and 28 was significantly lesser in Group $C$ than in Group S (Figure 1).

Watery stool was observed for 7 days post operation in Group C, but not in Group S.

\subsection{Quantitative RT-PCR}

Significant quantitative RT-PCR analyses enhanced
AQP8 mRNA expression in the ileum and remnant rectum of Group $C$ rats at days 7 ( $p<0.01)$; however, this was not observed at days 14 and 28 (Figure 2). In the ileum at days 7, the AQP8 mRNA expression level of Group $C$ was almost 4 times that of Group S. In the remnant rectum, it was almost 3 times that of Group S. The level, however, returned to normal at days 14 and 28.

\subsection{Immunohistochemistry}

Immunohistochemistry was used to detect the AQP8 protein expression in the apical membrane of the ileum in Group C and the apical membrane of the remnant rectum in both groups (Figure 3 ). In the ileum of Group $S$, no protein expression was detected. AQP8 mRNA expression in the ileum was low at days 14 and 28, but AQP8 protein in the ileum was maintained through the experimental period.

\subsection{Morphological Measurements}

The villus length of the ileum and crypt depth of the ileum and rectum were measured. The results are shown in Table 1. The villus length of the ileum on days 7 and 14 of Group $C$ was significantly greater than that of Group $\mathrm{S}$ ( $\mathrm{p}<0.002$ and 0.02 , respectively). The crypt depth remained unchanged (Table 2 ).

\section{DISCUSSION}

Since the discovery of AQP1, 12 mammalian AQPs have been recognized, and the clinical or physiological importance of AQPs has been shown or suggested; AQP0 mutations have been identified in some forms of congenital cataracts [14]. AQP1-null individuals have shown limited ability to maximally concentrate urine under water-deprived conditions [15] and have shown decreased pulmonary vascular permeability [16]. AQP2 mutations

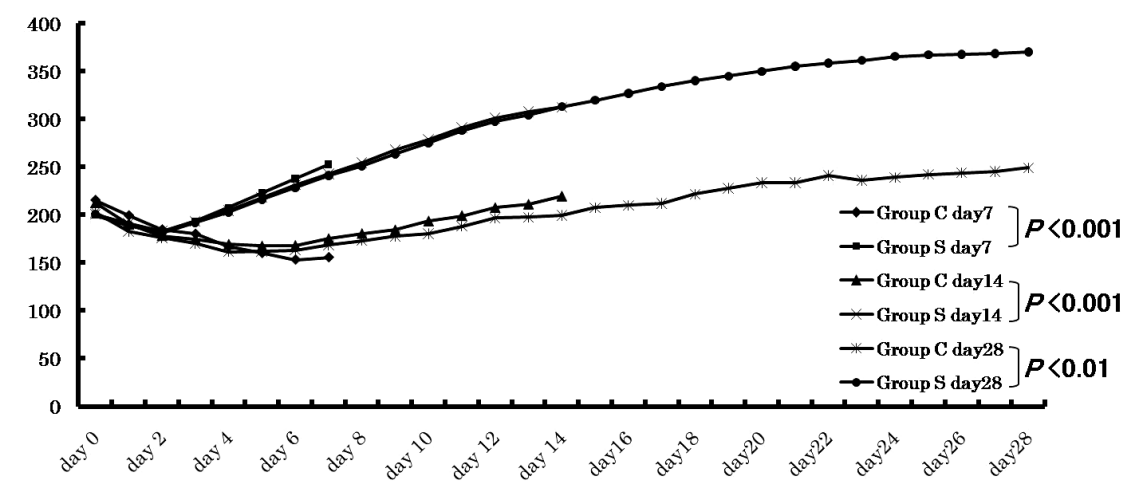

Figure 1. Weight of experimental animals. The rats in Group C showed a temporary decrease in weight from $210.5 \pm 14.9 \mathrm{~g}$ to $165.8 \pm 20.0 \mathrm{~g}$ at 7 days post operation. Group S showed a temporary decrease from $200.2 \pm 8.2 \mathrm{~g}$ to $192.7 \pm 8.4 \mathrm{~g}$ at 3 days post operation. The weight on postoperative days 7, 14, and 28 were significantly lesser in Group C than in Group S (p $<0.001$ on days 7 and 14 , and $\mathrm{p}<0.01$ on day 28). 


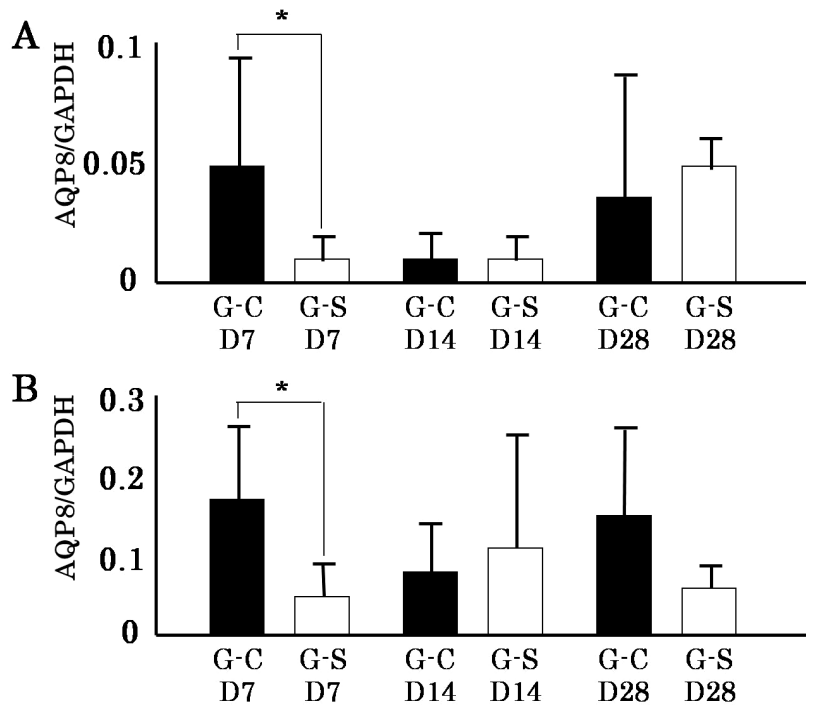

Figure 2. AQP8 mRNA expression. AQP8 mRNA expression in the ileum (A). B, AQP8 mRNA expression in the remnant rectum (B). AQP8 mRNA expression was enhanced in the ileum and the remnant rectum of Group $\mathrm{C}$ rats at day 7 ( $^{*} \mathrm{p}<$ $0.01)$.
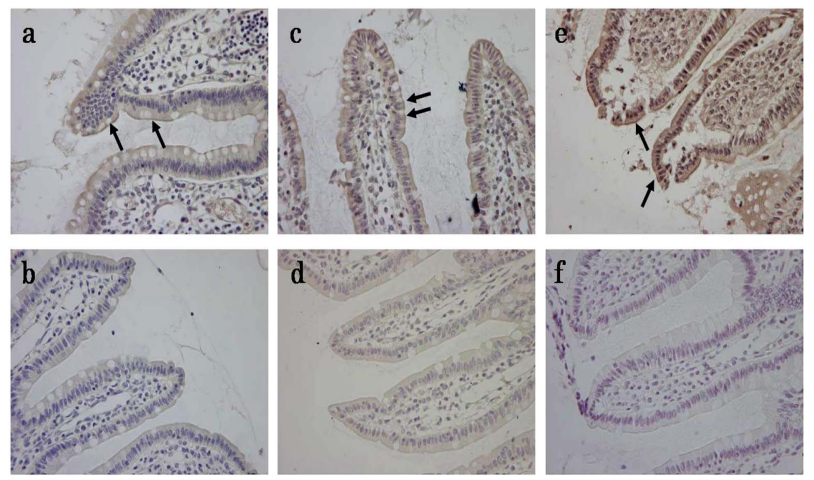

(A)
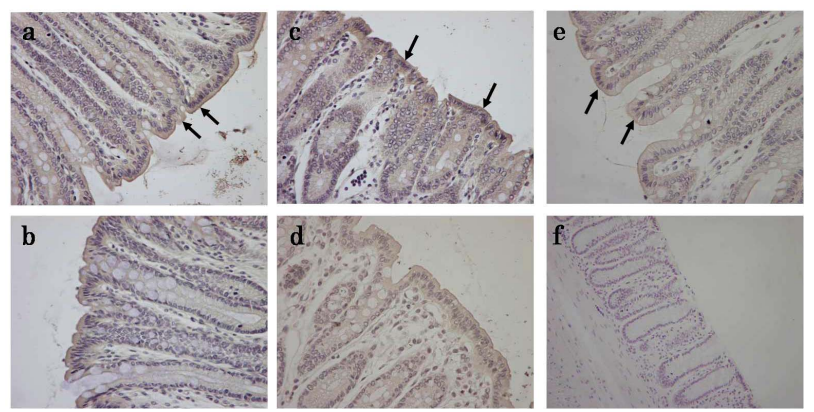

(B)

Figure 3. AQP8 protein expression. A, AQP8 protein expression in the ileum (A). AQP8 protein expression in the remnant rectum (B). AQP8 protein expression (arrow) was detected in the apical membrane of the ileum of Group $C$ and the apical membrane of the remnant rectum of both groups. The top line (a, c, e) is a specimen from Group C and the bottom line (b, d, f), from Group $\mathrm{S}$. The right column $(\mathrm{a}, \mathrm{b})$ is day 7 ; the middle column (c, d), day 14; and the left column (e, f), day 28. cause clinical congenital diabetes insipidus [17], and AQP3 and AQP4 seem to play a role in urinary concentration in knockout mice studies $[18,19]$. Abnormalities in AQP5 distribution have been shown to be related to Sjogren's syndrome [20]. Although several experimental models using transgenic mice lacking AQPs have been examined previously [21], the functions of AQPs in digestive organs remain unclear.

Previously, we have shown the expression, distribution, and localization of AQPs in rat digestive organs, including the GI tract: Generalized expression of AQP1 and AQP3 mRNA is observed widely along the gastrointestinal tract; AQP4 mRNA is expressed selectively in the lower portion of the stomach and small intestine; and AQP8 mRNA is expressed more selectively in the jejunum and colon [12,13]. Recently we cloned AQP10 from the human jejunum. AQP10 was selectively expressed in the upper sites of the small intestine, such as the duodenum and jejunum in humans [11]; however, AQP10 has not been identified yet in rats.

Purdy et al. [22] reported a change in AQP3 expression in the ileostomy model. We have previously shown enhanced expression of AQP8 mRNA after colectomy in rats. Here, we decided to construct a rat colectomy model to elucidate the function of AQP8 and intestinal adaptation.

We performed a quantitative investigation using RealTime PCR to elucidate the changes in AQP family gene expression of mRNA in the rat gastrointestinal tract after bowel removal. Our result revealing a prominent enhancement in AQP8 mRNA expression in the ileum and remnant rectum of the subtotal colectomy rats suggest that AQP8 in the ileum and remnant rectum is useful for water absorption.

We also used immunohistochemistry to confirm the enhancement of the AQP8 protein in the rectum of the subtotal colectomy rats being in accordance with the enhancement of AQP8 mrnaexpression. Our results showed prominent AQP8 protein on the columnar epithelial cells of the ileum and the remnant rectum of the subtotal colectomy rats; in the sham-operated rats, the staining was negligible. However, AQP8 mrnaexpression was normalized on days 14 and 28, suggesting that AQP8 protein expression in Group $C$ was enhanced even on days 28. This suggests that the half-life of the AQP8 protein would continue after a decrease in AQP8 mRNA expression, and the AQP8 protein would function for water absorption.

Morphologic measurements showed an elongated villus length and crypt depth of the ileum on days 7 and 14 in Group C. On days 28, there was no significant difference in the villus length between the groups. No significant difference was observed in the crypt depth of the rectum. Willis et al. [23] reported an increased villus 
Table 1. Villus length and crypt depth of ileum.

\begin{tabular}{ccccccc}
\hline & \multicolumn{2}{c}{ Day 7 } & & Day 14 & & Day 28 \\
\cline { 2 - 7 } & Group C & Group S & Group C & Group S & Group C & Group S \\
\hline Villus $(\mu \mathrm{m})$ & $345.1 \pm 36.7^{* *}$ & $265.4 \pm 36.3^{* *}$ & $357.2 \pm 112.8^{* *}$ & $240.9 \pm 143.3^{* *}$ & $346.9 \pm 53.9$ & $307.4 \pm 37.7$ \\
Crypt $(\mu \mathrm{m})$ & $193.6 \pm 41.7^{*}$ & $131.7 \pm 21.8^{*}$ & $208.3 \pm 50.2^{*}$ & $154.5 \pm 34.1^{*}$ & $203.4 \pm 42.9$ & $157.6 \pm 29.8$ \\
\hline
\end{tabular}

Group C, subtotal colectomized rat; Group S, sham operated rat; ${ }^{* *} \mathrm{p}<0.005$; ${ }^{*} \mathrm{p}<0.05$.

Table 2. Crypt depth of rectum.

\begin{tabular}{|c|c|c|c|c|c|c|}
\hline & \multicolumn{2}{|c|}{ Day 7} & \multicolumn{2}{|c|}{ Day 14} & \multicolumn{2}{|c|}{ Day 28} \\
\hline & Group C & Group S & Group C & Group S & Group C & Group S \\
\hline Crypt $(\mu \mathrm{m})$ & $315.7 \pm 47.8$ & $309.1 \pm 53.5$ & $305.6 \pm 73.1$ & $290.0 \pm 29.8$ & $303.9 \pm 47.4$ & $283.5 \pm 34.6$ \\
\hline
\end{tabular}

Group C, subtotal colectomized rat; Group S, sham operated rat.

length and density after colectomy in rats. They concluded that the increased ileal mucosal surface is probably responsible for the elevation in electrolyte and glucose absorption. Our results suggested that the elongated villi would assist AQP8 in water absorption in the subtotal colectomy model. We considered that most part of the adaptation of the ileum would be completed after 14 days.

Continuous diarrhea and weight loss were observed in Group C. The diarrhea continued and the bodyweight continued to decrease until 7 days after the operation, possibly suggesting that bodyweight loss and/or diarrhea would accelerate the adaptation of the intestine after colectomy in rats.

In summary, in the ileum and rectum in Group C, AQP8 mRNA expression was enhanced on days 7 and AQP8 protein expression was enhanced on days 7, 14 and 28. The villus length of the ileum was increased on days 7 and 14 in Group C. These results suggested that intestinal adaptation after subtotal colectomy occurred after 7 postoperative days.

\section{CONCLUSIONS}

The physiological roles of water channels in water transportation through the epithelial cell layer in the GI tract remain unclear. However, our results suggest an important role of AQP8 in the maintenance of intestinal fluid balance, and for the adaptation to postoperative conditions.

AQP8 may facilitate water movement through the epithelium of the ileum and the remnant rectum after subtotal colectomy.

\section{REFERENCES}

[1] King, L.S. and Agre, P. (1996) Pathophysiology of the aquaporin water channels. Annual Review of Physiology, 58, 619-648. doi:10.1146/annurev.ph.58.030196.003155

[2] Preston, G.M., Carrol, T.P., Guggino, W.B. and Agre, P. (1992) Appearance of water channels in Xenopus oocytes expressing red cell CHIP28 protein. Science, 256, 385387. doi:10.1126/science.256.5055.385

[3] Fushimi, K., Uchida, S., Hara, Y., Hirata, Y., Marumo, F. and Sasaki, S. (1993) Cloning and expression of apical membrane water channel of rat kidney collecting tubule. Nature, 361, 549-552. doi:10.1038/361549a0

[4] Ishibashi, K., Sasaki, S., Fushimi, K., Uchida, S., Kuwahara, M., Saito, H., Furukawa, T., Nakajima, K., Yamaguchi, Y. and Marumo, F. (1994) Molecular cloning and expression of a member of the aquaporin family with permeability to glycerol and urea in addition to water expressed at the basolateral membrane of kidney collecting duct cells. Proceeding of the National Academy of Sciences of the United States of America, 91, 6269-6273.

[5] Jung, J.S., Bhat, R.V., Preston, G.M., Guggino, W.B., Baraban, J.M. and Agre, P. (1994) Molecular characterization of an aquaporin cDNA from brain candidate osmoreceptor and regulator of water balance. Proceeding of the National Academy of Sciences of the United States of America, 91, 13052-13056.

[6] Raina, S., Preston, G.M., Guggino, W.B. and Agre, P. (1995) Molecular cloning and characterization of an aquaporin cDNA from salivary, lacrimal and respiratory tissues. Journal Biological Chemistry, 270, 1908-1912. doi:10.1074/jbc.270.4.1908

[7] Ma, T., Yang, B., Kuo, W.L. and Verkman, A.S. (1996) cDNA cloning and gene structure of a novel water channel expressed exclusively in human kidney: Evidence for a gene cluster of aquaporins at chromosome locus 12q13. Genomics, 35, 543-550. doi:10.1006/geno.1996.0396

[8] Ishibashi, K., Kuwahara, M., Gu, Y., Kageyama, Y., Tohsaka, A., Suzuki, F., Marumo, F. and Sasaki, S. (1997) Cloning and functional expression of a new water channel abundantly expressed in the testis permeable to water, glycerol and urea. Journal Biological Chemistry, 272, 20782-20786. doi:10.1074/jbc.272.33.20782 
[9] Koyama, Y., Yamamoto, T., Kondo, D., Funaki, H., Yaoita, E., Kawasaki, K., Sato, N., Hatakeyama, K. and Kihara, I. (1997) Molecular cloning of a new aquaporin from rat pancreas and liver. Journal Biological Chemistry, 272, 30329-30333. doi:10.1074/jbc.272.48.30329

[10] Ishibashi, K., Kuwahara, M., Gu, Y., Tanaka, Y., Marumo, F. and Sasaki, S. (1998) Cloning and functional expression of a new aquaporin (AQP9) abundantly expressed in the peripheral leukocytes permeable to water and urea, but not to glycerol. Biochemical and Biophysical Research Communications, 244, 268-274. doi:10.1006/bbrc.1998.8252

[11] Hatakeyama, S., Yoshida, Y., Tani, T., Koyama, Y., Nihei, K., Ohshiro, K., Kamiie, J.I., Yaoita, E., Suda, T., Hatakeyama, K. and Yamamoto T. (2001) Cloning of a new aquaporin (aqp10) abundantly expressed in duodenum and jejunum. Biochemical and Biophysical Research Communications, 287, 814-819. doi:10.1006/bbrc.2001.5661

[12] Koyama, Y., Yamamoto, T., Tani, T., Nihei, K., Kondo, D., Funaki, H., Yaoita, E., Kawasaki, K., Sato, N., Hatakeyama, K. and Kihara I. (1999) Expression and localization of aquaporins in rat gastrointestinal tract. American Journal of Physiology, 276, C621-C627.

[13] Tani, T., Koyama, Y., Nihei, K., Hatakeyama, S., Ohshiro, K., Yoshida, Y., Yaoita, E., Sakai, Y., Hatakeyama, K. and Yamamoto, T. (2001) Immunolocalization of aquaporin-8 in rat digestive organs and testis. Archives of Histology and Cytology, 64, 159-168. doi:10.1679/aohc.64.159

[14] Berry, V., Francis, P., Kaushal, S., Moore, A. and Bhattacharyta, S. (2000) Missense mutations in MIP underlie autosomal dominant polymorphic and lamellar cataracts linked to 12q. Nature Genetics, 25, 15-17. doi:10.1038/75538

[15] King, L.S., Choi, M., Fernandez, P.C., Cartron, J.P. and Agre, P. (2001) Defective urinary-concentrating ability due to a complete deficiency of aquaporin-1. New England Journal of Medicine, 345, 175-179. doi:10.1056/NEJM200107193450304

[16] King, L.S., Nielsen, S., Agre, P. and Brown, R.H. (2002)
Decreased pulmonary vascular permeability in aquaporin1-null humans. Proceeding of the National Academy of Sciences of the United States of America, 99, 1059-1063.

[17] Deen, P.M., Verdijk, M.A., Knoers, N.V., Wieringa, B., Monnens, L.A., van Os, C.H. and van Oost, B.A. (1994) Requirement of human renal water channel aquaporin-2 for vasopressin-dependent concentration of urine. Science, 264, 92-95. doi:10.1126/science.8140421

[18] Ma, T., Song, Y., Yang, B., Gillespie, A., Carlson, E.J., Epstein, C.J. and Verkman, A.S. (2000) Nephrogenic diabetes inspidus in mice lacking aquaporin-3 water channels. Proceeding of the National Academy of Sciences of the United States of America, 97, 4386-4391.

[19] Ma, T., Yang, B., Gillespie, A., Carison, E.J., Epstein, C.J. and Verkman, A.S. (1997) Generation and phenotype of a transgenic knockout mouse lacking the mercurialinsensitive water channel aquaporin-4. The Journal of Clinical Investigation, 100, 957-962. doi:10.1172/JCI231

[20] Steinfeld, S., Cogan, E., King, L.S., Agre, P., Kiss, R. and Delporte, C. (2001) Abnormal distribution of aquaporin-5 water channel protein in salivary glands from Sjogren's syndrome patients. Laboratory investigation, 81, 143148. doi:10.1038/labinvest.3780221

[21] Verkman, A.S., Yang, B., Song, Y., Manley, G.T. and Ma, T. (2000) Role of water channels in fluid transport studied by phenotype analysis of aquaporin knockout mice. Experimental physiology, 85, 233S-241S. doi:10.1111/j.1469-445X.2000.tb00028.x

[22] Purdy, M.J., Cima, R.R., Doble, M.A., Klein, M.A., Zinner, M.J. and Soybel, D.J. (1999) Selective decrease in levels of mRNA encoding a water channel (AQP3) in ileal mucosa after ileostomy in the rat. Journal of Gastrointestinal Surgery, 3, 54-60. doi:10.1016/S1091-255X(99)80009-2

[23] Willis, S., Kisielinski, K., Klosterhalfen, B. and Schumpelick, V. (2002) Morphological and functional adaptation of the small intestine after colectomy and ileal pouch-anal anastomosis in rats. International Journal of colorectal Disease, 17, 85-91. doi:10.1007/s003840100352 